\title{
Itinéraire ethnographique d'une doctorante : dialogue autour de la (dé)construction de l'objet de recherche
}

\author{
Marjorie Vidal, Doctorante
}

Université de Montréal

Joëlle Morrissette, Ph.D.

Université de Montréal

\begin{abstract}
Résumé
Cet article rend compte des manières dont la vigilance ethnographique a permis de composer avec les obstacles qu'une doctorante - ethnographe en herbe - a rencontrés dans la construction de son objet de recherche. La réflexion proposée ici s'appuie ainsi sur sa recherche doctorale qui porte sur les processus relationnels mobilisés par des élèves de milieux défavorisés pour «s'en sortir ». La trame narrative prend la forme d'une conversation entre la doctorante et sa codirectrice et permet de retracer les différentes épreuves, hésitations et négociations qui se sont soldées en transformations successives de l'objet ethnographique. Nous montrerons ainsi qu'au fil de rencontres, la vigilance ethnographique permet de se détacher des cadres formels prescrits, théoriques et méthodologiques, et d'exploiter les contraintes du terrain en ressources au profit de la construction ancrée de l'objet.

Mots clés

PERSPECTIVE INTERACTIONNISTE, ETHNOGRAPHIE, RÉSEAU MUTUEL D'INFLUENCES, CONSTRUCTION PROGRESSIVE DE L'OBJET
\end{abstract}

\section{Introduction}

En quoi la vigilance ethnographique permet-elle de négocier les obstacles que l'ethnographe rencontre dans la construction de son objet de recherche? Pour répondre à cette question, nous proposons dans cet article une « incursion ethnographique » qui prend la forme d'une randonnée sans boussole, remplie de pertes de sens épistémologiques, de glissements de terrain théoriques, d'impasses et de bifurcations méthodologiques. Pourtant, nous prétendons que c'est au contact de ces « aspérités » que s'affine la vigilance de

RECHERCHES QUALITATIVES - Vol. 33(1), pp. 86-108.

VIGILANCE ETHNOGRAPHIQUE ET RÉFLEXIVITÉ MÉTHODOLOGIQUE

ISSN 1715-8702 - http://www.recherche-qualitative.qc.ca/Revue.html

(C) 2014 Association pour la recherche qualitative 
l'ethnographe. L'objectif de cet article est donc de théoriser ces phénomènes ou processus qui participent de la définition de l'objet ethnographique à partir de l'éclairage de la notion de vigilance. La définition de la vigilance que nous proposons correspond donc à cette idée d'itinéraire chaotique en terre inconnue. Elle se manifeste quand l'ethnographe rencontre des embûches sur son terrain et lui permet d'appréhender les obstacles non plus comme des contraintes, mais comme des ressources qui vont transformer successivement l'objet de recherche. Confronté à ces difficultés, l'ethnographe vigilant doit accepter de perdre le nord pour mieux se réorienter et redéfinir ses perspectives théoriques et méthodologiques.

Les différentes étapes de cet itinéraire ethnographique sont illustrées à partir d'une recherche doctorale (Vidal, en cours), dont la démarche repose sur une ethnographie scolaire qui a débuté en 2009 et a duré trois ans. Elle s'est déroulée dans une école secondaire d'un quartier de Montréal à forte concentration multiethnique et recouvre plusieurs méthodes de collecte : analyse de documents officiels, observation participante, questionnaires, entretiens et analyses de groupe. Les participants sont des élèves âgés de 13 à 17 ans. L'hypothèse de départ de cette recherche est une idée relativement répandue, notamment en milieu «défavorisé ${ }^{1}{ }^{2}$ : l'idée que les relations sociales «comptent», c'est-à-dire qu'elles peuvent jouer un rôle compensatoire et permettre à certains élèves d'accéder à des ressources dont ils sont initialement dépourvus.

L'école permet à l'élève d'entrer en relation avec différents acteurs (enseignants, professionnels, administrateurs) qui peuvent l'aider quand il rencontre des difficultés dans son parcours éducatif. Or, certains élèves ne semblent pas mobiliser ces relations de la manière prévue par l'école. Cette recherche vise donc à mettre à jour les processus relationnels que les élèves mobilisent pour «s'en sortir» au cours de leur trajectoire d'élève. Nous privilégions en effet l'idée de «s'en sortir » qui dépasse la notion de réussite scolaire, car celle-ci est restreinte à la perspective normative de l'institution, à l'idée de performance scolaire (nous y revenons dans la section Déviation, lorsque nous abordons la rencontre avec les acteurs du terrain). Cette ethnographie est adossée à une perspective interactionniste (Morrissette, Guignon, \& Demazière, 2011); elle appréhende le fait de «s'en sortir » comme le résultat de multiples interactions entre l'élève et son environnement social.

Cet itinéraire s'ouvre sur une rencontre : les chemins d'une doctorante et d'une professeure se croisent autour de ce qui deviendra vite une préoccupation commune, soit les méthodes qualitatives en général et l'ethnographie en particulier. La définition de la vigilance que nous proposons 
est ainsi étroitement liée à cette idée de rencontre. Elle s'inspire en particulier des travaux d'Howard Becker en ce que nous appréhendons cette notion à partir des interactions et des réseaux d'influences mutuelles. C'est à l'instar de Chapoulie (2001) que nous conduisons l'argumentation dans cet article. Cet auteur retrace l'histoire de la tradition de Chicago à partir des influences entre professeurs et étudiants au département de sociologie de l'Université de Chicago, rejetant ainsi l'idée d'une école de pensée unique autour d'un certain cadre théorique.

Pour remonter la trame de ces interactions (Demazière, 2008; Morrissette, Guignon, \& Demazière, 2011) - et des errances ethnographiques -, nous choisissons une présentation sur le mode de la discussion. Fortin et Houssa (2012) ont déjà eu recours à ce type de trame narrative à travers une fiction en quatre actes sur des questions d'ordre théorique relatives à l'ethnographie. Dans une visée de théorisation progressive de l'objet de recherche qui s'appuie davantage sur la confrontation avec le terrain, le présent article propose plutôt de reconstituer a posteriori les principaux échanges entre la doctorante et la professeure qui ont porté sur les obstacles et errances ethnographiques. Il vise à théoriser les phénomènes qui ont affecté progressivement l'objet de recherche. À travers cette argumentation duale, nous donnons à voir notre conceptualisation de l'idée de vigilance en reconstituant ses différentes expressions: nous mettons ainsi en évidence les obstacles théoriques et empiriques ainsi que la manière dont l'argumentation duale a permis de les négocier. De fait, de nouvelles perspectives ont émergé au fur et à mesure. Elles se sont modifiées au contact du terrain et se sont traduites en transformations successives de l'objet de recherche. Et, parallèlement, le fait que l'objet se transforme a consolidé de nouvelles perspectives.

Le plan de l'argumentation suit la courbe de l'itinéraire. Aussi, après avoir problématisé la difficulté de développer la vigilance pour tout doctorant, nous proposons au lecteur de s'attarder sur trois des grandes étapes qui correspondent en fait à trois rencontres et transformations successives qui ont affecté l'objet de recherche. Nous concluons enfin sur la façon dont ces différentes rencontres permettent une construction théorique de la notion de vigilance.

\section{Problématique : de la difficulté à développer la vigilance pour une ethnographe en herbe}

Il existe pour tout ethnographe en herbe deux temps ${ }^{2}$ : le temps de l'institution, qui correspond au temps des unités de rattachement universitaire qui encadrent le cheminement doctoral, et le temps de l'ethnographie, qui est le temps du terrain. Or ces deux temps, réunis dans une même démarche ethnographique, ne 
procèdent pas de la même logique et tendent à être contradictoires, ce qui vient complexifier la démarche d'un étudiant au doctorat. C'est ce que nous proposons de discuter dans la partie suivante.

\section{Le temps de l'institution}

Marjorie : Quand j'ai commencé mon doctorat en 2009, j'ai rapidement compris que l'étudiant au doctorat en sciences de l'éducation au Québec doit être efficace et surtout efficient: il doit produire en fonction d'un cadre institutionnel et d'un cheminement qui lui est imposé dès son entrée dans les cycles supérieurs. De fait, il dispose d'un temps limité (deux ans) à travers lequel il doit passer par des étapes obligatoires et ordonnées (cours et séminaires), sanctionnées par des examens convenus par chacun des départements. Ces différentes étapes constituent l'avancement des chapitres et correspondent d'ailleurs à la structure classique de la présentation d'une thèse doctorale (la recension des écrits sur le domaine et sur l'objet; le cadre théorique; la méthodologie; l'analyse des données; l'interprétation des résultats). De nombreux enjeux économiques permettent d'expliquer cet encadrement strict de la gestion du temps. En outre, le système de bourses mis en place aux cycles d'enseignement supérieur encourage un cheminement rapide, qui ne s'étire pas. Il impose un certain rythme scolaire indépendamment des contextes des étudiants. À titre d'exemple, je reproduis dans le Tableau 1 un extrait d'échéancier que j'ai fourni, conformément aux exigences administratives, à la Faculté des études supérieures et postdoctorales (FESP) en 2011 dans le cadre d'un concours pour les bourses d'excellence.

Joëlle : Comme on peut le voir à travers ton échéancier, les exigences académiques se succèdent dans le cheminement de l'étudiant au doctorat et ces balises, qui circonscrivent dans le temps la collecte des données, exigent une productivité constante. Les contingences universitaires standardisent une certaine conception du temps qui doit être exploité selon les différentes exigences des unités académiques et optimisé en référence à un parcours standard d'un étudiant au doctorat (quatre ans). De fait, ces contingences s'accommodent mal de temps perdu et la période d'immersion de l'ethnographe se retrouve souvent réduite à la portion congrue : de septembre à décembre, si l'on tient compte de ton échéancier. D'ailleurs, cet échéancier sur lequel nous sommes revenues à plusieurs reprises et que l'on qualifiait d'«engrenage contraignant $»$, il semble que tu ne l'aies finalement pas respecté...

Marjorie : C'est vrai, de nombreux obstacles administratifs se sont dressés sur ma trajectoire et ont affecté les échéances de manière substantielle, de sorte qu'ils ont quelque peu allongé le calendrier. Tu dois probablement te rappeler les difficultés rencontrées lors de la réalisation du dossier pour le 
Tableau 1

Échéancier (FESP, 2011)

\begin{tabular}{|c|c|c|}
\hline \multirow[t]{3}{*}{$09 / 09-12 / 09$} & Définition del'objet d'étude & $\underline{\text { Objectifs du cours }}$ \\
\hline & Choix du directeur de thèse & ETA 7001 \\
\hline & Sélection de la documentation & \\
\hline \multirow[t]{2}{*}{$01 / 10-04 / 10$} & Maîtrise des concepts, approches, modèles théoriques. & ETA 7002 \\
\hline & $\begin{array}{l}\text { Formulation des questions préliminaires et des } \\
\text { objectifs }\end{array}$ & \\
\hline $05 / 10$ & Examen de synthèse & \\
\hline $09 / 10-12 / 10$ & $\begin{array}{l}\text { Rédaction de la partie cadre théorique avec } \\
\text { commentaires }\end{array}$ & ETA 7000 \\
\hline \multirow[t]{2}{*}{$01 / 11-04 / 11$} & Reprise et correction de la partie cadre théorique & ETA 7000 \\
\hline & $\begin{array}{l}\text { Choix et conception de la méthode de collecte de } \\
\text { données }\end{array}$ & ETA 6507 \\
\hline $05 / 11$ & Rédaction et dépôt du certificat d'éthique & \\
\hline \multirow[t]{3}{*}{$06 / 11-08 / 11$} & \multicolumn{2}{|c|}{ Recherches du terrain et prises de contact avec les gens du milieu } \\
\hline & \multicolumn{2}{|c|}{ Sélection des participants } \\
\hline & \multicolumn{2}{|l|}{ Approbation des parents d'élèves } \\
\hline $09 / 11-12 / 11$ & \multicolumn{2}{|l|}{ Collecte de données } \\
\hline $01 / 12-03 / 12$ & \multicolumn{2}{|l|}{ Analyse des données } \\
\hline $05 / 12-08 / 12$ & \multicolumn{2}{|l|}{ Rédaction et dépôt de la thèse } \\
\hline \multicolumn{3}{|c|}{ Soutenance } \\
\hline
\end{tabular}

Comité d'éthique, qui est devenue un véritable parcours du combattant puisque la forme institutionnelle des documents éthiques (lettres de consentement des parents d'élèves mineurs, de présentation du projet) a rebuté la majorité des parents dont il me fallait obtenir le consentement. Je rappelle ici que mon ethnographie s'est déroulée dans une école secondaire de milieu "défavorisé » et à forte concentration multiethnique. Plusieurs parents étaient analphabètes, ne parlaient pas bien le français, n'étaient pas en règle avec leur visa de séjour au Canada ou enfin ne comprenaient pas ce qu'on attendait d'eux et de leurs enfants. Coulon (1997) dirait qu'ils faisaient l'expérience de l'«étrangeté culturelle».

Cette différence de culture entre la rigidité, les balises du milieu universitaire et la souplesse induite par mon terrain de recherche est le premier obstacle qu'il m'a fallu franchir pour avoir le droit d'interviewer les participants. Becker (2011) y fait écho quand il met l'accent sur les obstacles administratifs auxquels se heurtent les chercheurs. Il critique notamment 
l'institutionnalisation des règles d'éthique et la normalisation des exigences institutionnelles que l'étudiant finit par appréhender comme un passage obligatoire dans sa scolarité. Dans mon cas et en dépit de ces exigences administratives, le Comité d'éthique s'est montré conciliant et a finalement accepté d'autres modes de validation plus appropriés. J'ai ainsi pu valider les questionnaires par téléphone avec les parents en présence de l'élève, d'un adulte et parfois d'un traducteur.

Joëlle : Cause ou conséquence de ces exigences administratives strictes de l'université que, pour certaines, tu as détaillées, la culture ethnographique n'est pas très développée en sciences de l'éducation au Québec, contrairement à certaines disciplines comme l'anthropologie ou la sociologie qui ne remettent en question ni son intérêt ni sa rigueur méthodologique. Dans le domaine de l'éducation, on est encore confronté à une certaine forme de méconnaissance quant à la contribution de l'ethnographie et à de nombreuses réticences concernant sa mise en œuvre. Les apports de l'ethnographie au corpus de la recherche scientifique contemporaine sont pourtant indéniables: elle a notamment permis de renouveler les interprétations et de comprendre des problématiques actuelles complexes et confère une place privilégiée aux acteurs sociaux. Déjà en 1987, Derouet et Henriot évoquaient la pertinence d'instruments de connaissances à échelle d'action des acteurs sociaux. Cette même voie est empruntée par Woods (1990) dans son Ethnographie de l'école lorsqu'il analyse comment les enseignants comprennent et construisent leurs pratiques dans l'interaction.

Marjorie : Je me souviens de la présentation d'un invité dans ton cours : sans remettre en cause la fécondité de la démarche ethnographique, Poupart $(2011)^{3}$ avait expliqué ses réticences à laisser partir ses étudiants sur leur terrain sans préparation au préalable. Ces réticences, je les ai souvent ressenties chez d'autres chercheurs. Elles seraient peut-être attribuables au fait que l'ethnographie réclame un encadrement exigeant et appelle une certaine capacité à pouvoir vivre avec l'incertitude du parcours. Pour certains, ce choix méthodologique est chronophage, une «mode de hippie». Pour d'autres, l'induction de la démarche reviendrait à rejeter le corpus théorique déjà existant. Bref, pour reprendre une expression de Vienne (2005), l'ethnographie «fait peur $»$.

\section{Le temps de l'ethnographie}

Joëlle : De fait, cette rationalisation du temps et ce cadrage universitaire strict vont à l'encontre de la démarche ethnographique qui vit mal les restrictions temporelles à horizon prédéfini. Comme nous tendons à le démontrer, les errances et errements sont primordiaux pour développer la vigilance et la 
réflexivité méthodologiques de l'étudiant. Ainsi, ce temps perdu du point de vue de la rentabilité institutionnelle, cette étape de créativité non instrumentalisée, bref ce que l'on peut appeler le temps ethnographique a été nécessaire pour que tu puisses développer ta «sensibilité au terrain» (Becker, 1982/2006). Et c'est cette sensibilité qui va permettre à l'ethnographe d'être attentif aux « impondérables de la vie authentique », dirait Malinowski (1922, pp. 75-76).

Marjorie : Aujourd'hui, je me rends compte de la fécondité de cette période, de ce temps perdu à traîner çà et là dans les couloirs de l'école au cours de mes observations, à approcher les acteurs de mon terrain de recherche, à apprivoiser le contexte et m'imprégner des lieux. J'étais, pour reprendre la métaphore de Soukup (2012), «l'ethnographe-flâneur » de Baudelaire et Walter Benjamin. Et c'est notamment au cours de cette flânerie, en partie orchestrée, que se sont révélées les prémices de ma recherche. Dans mes pérégrinations, je retrouvais une idée de manière relativement répandue : les relations sociales comptent, c'est-à-dire qu'elles peuvent jouer un rôle compensatoire et favoriser la réussite des élèves qui présentent des conditions initiales défavorables.

Je me souviens avoir plusieurs fois parlé avec toi du fait que quand on commence une ethnographie, c'est comme si le regard n'était pas «armé », c'est-à-dire pas instruit théoriquement. Parallèlement à mon terrain, il a donc fallu que je développe une connaissance générale des écrits sur les inégalités sociales et scolaires. Je me suis donc intéressée aux différents courants théoriques qui permettent d'appréhender ce phénomène, des approches déterministes comme celles des fonctionnalistes (Parsons, 1959) ou des marxistes (Baudelot \& Establet, 1971) et des structuralistes (Bourdieu \& Passeron, 1970), jusqu'aux approches qui ciblent davantage l'individu et son pouvoir d'action comme l'individualisme méthodologique (Boudon, 1973) et l'interactionnisme symbolique (Barakett \& Cleghorn 2008). Je retrouvais également cette idée des relations compensatoires chez certains canons du domaine tels que Zhang, DeBlois, Kamanzi et Deniger (2008) qui soulignent l'importance des relations sociales dans la réussite des élèves de milieux défavorisés.

Joëlle : Et cette connaissance a priori n'a pas formaté ton objet de recherche. Sans pour autant tomber dans une démarche d'induction pure, la tabula rasa préconisée par Glaser et Strauss (1967) où tout part du terrain, l'ethnographe jongle effectivement en permanence avec la théorie et ce qu'il découvre sur le terrain. Comme le souligne Blumer (1969), les concepts se construisent et se transforment progressivement, avec le temps; ils sont susceptibles d'être modifiés dans l'acte de recherche, car inscrits dans des processus qui les font évoluer. L'instruction théorique doit donc passer par une 
théorisation progressive de l'objet sur le terrain; l'ethnographe a beau échafauder des objets de recherche, plusieurs d'entre eux seront invalidés par le terrain (Tillard, 2011). La théorie ne doit pas verrouiller les choix méthodologiques par avance et l'ethnographe a avantage à garder une ouverture pour la redéfinition de son objet de recherche. Ici, la vigilance est primordiale et requiert d'être attentive à des changements infinitésimaux. C'est ainsi que ton objet de recherche a subi dans le temps plusieurs modifications.

\section{Appel à la vigilance sur la route ethnographique}

En conclusion de cette première partie, nous proposons un premier pas vers notre acception théorique de la vigilance. Celle-ci s'acquiert et s'aiguise donc au fil du temps, avec l'immersion, au contact du terrain (mais sans négliger les lectures), donc en «vivant» son ethnographie. En dépit des obstacles, cette vigilance s'est particulièrement manifestée - jusqu'à devenir une nécessité lors des moments critiques et a été rendue possible grâce à différentes rencontres qui ont jalonné le cheminement doctoral. Comme nous l'avons déjà dit, la définition de la vigilance que nous proposons dans cet article est étroitement liée à ces rencontres et à l'idée de réseaux mutuels d'influence (Becker, 1982/2006). La vigilance, c'est donc se balader - et accepter de «se faire balader »-, mais c'est également savoir remettre en question les a priori sur l'objet pour pouvoir intégrer de nouvelles perspectives. C'est ce que nous développons dans la partie suivante.

\section{Les rencontres}

Nous proposons une discussion autour de trois grandes étapes de ce parcours un peu chaotique qui correspondent à trois rencontres qui ont affecté le processus de définition de l'objet de recherche par l'intégration de nouvelles perspectives épistémologiques/théoriques et méthodologiques. Les trois plus marquantes sont retenues : la première est la rencontre avec une des auteures de cet article - qui allait devenir la codirectrice de recherche -, qui s'est traduite par une transformation de l'objet théorique; la deuxième est la rencontre avec les acteurs du terrain, qui a entraîné une modification de l'objet empirique; la troisième est la rencontre avec soi-même (en tant qu'ethnographe) et avec les participants de la recherche, qui a conduit à une distanciation par rapport au scénario méthodologique initial.

Dans la prolongation de la métaphore filée, chaque partie se présente de la même manière: la section $\grave{A}$ rebrousse-chemin conduit vers les obstacles théoriques et empiriques qui se sont dressés sur le parcours de l'étudiante, tandis que L'Aire de repos et de réflexion restitue les échanges entre la doctorante et la professeure qui ont permis de négocier ces obstacles. La Déviation retrace l'exploitation des nouvelles perspectives qui se sont traduites in fine en 
transformations successives de l'objet de recherche. Enfin, L'Appel à la vigilance sur la route ethnographique reprend le propos sous l'éclairage de la vigilance.

\section{La rencontre avec la codirectrice}

En cours de route, la doctorante est partie suivre, par curiosité et un peu par hasard, un cours de méthodologies qualitatives donné par la coauteure de cet article (ETA6507, Démarches d'investigation des pratiques), devenue par la suite codirectrice de ses études. Leur rencontre est le premier jalon du parcours de l'ethnographe en devenir. Cette rencontre s'est soldée par un bouleversement de perspectives théoriques qui a affecté l'objet de recherche initial (les relations sociales) jusqu'à ce qu'il se modifie (les processus relationnels).

\section{À rebrousse-chemin}

Marjorie : Quand j'ai commencé ton cours, je portais un regard naïf sur mon terrain en accord avec la démarche qu'on m'avait jusqu'alors enseignée dans mes autres cours universitaires. Ma grille de lecture était préstructurée, c'est-àdire que mon objet de recherche initial (les relations sociales) était déjà formaté en fonction des grandes catégories issues de travaux de recherche. Dans cette optique, et en conformité avec un parcours professionnel plus économique et statistique, mes perspectives épistémologiques s'ancraient dans une approche quantitative et hypothético-déductive. J'envisageais de «tester» une hypothèse préétablie: les liens entre les relations sociales (variables dépendantes) et la réussite scolaire (variable indépendante). J'avais pour objectif de mesurer l'importance de certaines relations sociales dans la réussite (scolaire) des élèves, notamment à partir de questionnaires sur les réseaux sociaux. À cette époque, j'appréhendais l'ethnographie comme un prétexte, une étape préliminaire à ma collecte pour pouvoir approcher les élèves. Le concept de capital social me permettait de catégoriser les relations entre les différents acteurs, à savoir les parents, l'école et la communauté. Yan (1999), Dika et Singh (2002) ou encore Coleman (1988), des auteurs-clés du domaine, font état d'une liste de relations entre les différents acteurs, certaines plus significatives que d'autres. Je m'apprêtais donc à valider ou infirmer ces différentes relations causales dans le cadre de ma propre recherche.

Joëlle : Je me souviens qu'au tout début du cours, tu pensais qu'il n'y avait qu'une seule manière de faire la recherche; je m'étais alors donné pour mission d'ébranler un peu tes certitudes! Dans mon cours, tu t'es ouverte à d'autres perspectives, découvrant tout un pan de la recherche que tu ne connaissais pas jusque-là : l'apport des méthodes qualitatives et leur versant interprétatif. En levant le voile sur cette autre façon de poser les problèmes de recherche, ce cours a, d'une certaine manière, renversé tes perspectives 
épistémologiques et, alors que tu arpentais ton terrain, tu t'es progressivement rendu compte des limites de ton cadre théorique (le capital social) pour appréhender ton objet, les relations sociales. Mais cette transformation n'a pas été radicale et je me souviens avoir semé sur ton parcours ce qu'un professeur membre de mon propre comité de thèse il y a quelques années - appelait des «petits obstacles sympathiques », c'est-à-dire des embûches qui obligent à se questionner, à développer une réflexivité critique sur le processus de production de connaissances. Au fil des séances, je t'ai ainsi vue t'interroger, être déstabilisée, interpellée, etc.

Aire de repos et de réflexion

Marjorie : En effet, à mesure que j'intégrais des nouvelles perspectives épistémologiques/théoriques proposées, j'avais un peu l'impression que mon projet me conduisait à «faire entrer des carrés dans des ronds », si je puis dire, ou, pour reprendre l'image de Blumer (1969), forçait les données émergentes à entrer dans un moule théorique préétabli. Le terrain me faisait l'effet d'être réfractaire et j'étais confrontée à des données « résistantes », « rebelles ». Pour donner un exemple, alors que Yan (1999) et Coleman (1988) insistent sur l'importance de la relation entre l'enseignant et les parents pour la réussite des élèves, mes observations m'amenaient plutôt à comprendre que lorsque le parent rencontrait l'enseignant, c'était généralement synonyme de punition ou de pression sur l'élève ${ }^{4}$, et donc loin de contribuer à sa réussite.

Bref, le terrain présentait ainsi une vision beaucoup plus complexe et dynamique qu'elle s'était offerte à moi dans un premier temps. Je me suis retrouvée dans une impasse avec mon terrain. Mes perspectives commençaient à ralentir ma progression. Elles perdaient du sens par rapport à la posture plus respectueuse du terrain que je développais, inspirée par la démarche des auteurs associés à la tradition de Chicago, qui s'approprient, interprètent et traduisent une perspective interactionniste dans des opérations de recherche et des espaces disciplinaires variés. Mon cadre théorique était devenu un fardeau. J'étais donc un peu perdue, et c'est à ce moment-là que tu m'as introduite à de nouveaux appuis théoriques.

Joëlle : Un peu comme un professeur m'avait fait cadeau de Becker, je t'ai alors présentée à Giddens (1984), comme une suggestion de lecture « innocente », comme on lance un ver à l'eau en espérant que le poisson saute dessus pour le dévorer... Je voyais la fécondité des liens à faire entre sa théorie de la structuration et ton objet de recherche, et l'intérêt de reconnaître, à sa suite, le caractère réflexif et orienté des actions, soit exactement ce que tu me rapportais après chacune de tes explorations sur le terrain. En fait, tu découvrais des élèves beaucoup moins passifs et victimes du milieu socioculturel défavorisé 
que tes premiers appuis théoriques t'invitaient à considérer. En d'autres mots, ces jeunes que tu apprenais à connaître sur le terrain de ta recherche, et dont tu me parlais, me semblaient être «compétents», au sens où l'entend Giddens, c'est-à-dire qu'ils jouaient de leur marge de manœuvre, disposaient de ressources pour agir et se montraient réflexifs par rapport à cet agir lorsque tu les sollicitais dans cette perspective.

\section{Déviation}

Marjorie : Je me suis trouvée rapidement des affinités intellectuelles avec Giddens, notamment en raison de la souplesse de son cadre théorique qui se veut plus une vision générale du monde social, une perspective pour appréhender mon objet, qu'un modèle structuré par des concepts articulés entre eux, telles des boîtes qui l'auraient fragmenté en dimensions. Dans cette optique, sa théorie me semblait mieux convenir à une démarche ethnographique progressive. Elle se démarque des approches structuralistes d'auteurs comme Coleman (1988) ou Bourdieu et Passeron (1970), qui limitent en quelque sorte la possibilité d'action des individus et rendent ainsi plus difficile de concevoir que les élèves puissent «s'en sortir ». La théorie de la structuration de Giddens (1984) ${ }^{5}$ m'a également permis d'orienter le regard que je portais sur le terrain de manière non prescriptive et de dépasser la simple opposition entre l'acteur et la structure. En effet, pour van Haecht (1998), Giddens met en relation les conduites orientées des acteurs avec des règles et des ressources qui relèvent d'un contexte plus large que celui de l'interaction immédiate: acteurs et systèmes sont des «doubles en soi ».

Joëlle : C'est donc le regard guidé par ces nouveaux appuis théoriques dans le sens de sensibilisé et non dans le sens de formaté - que tu as ensuite décidé de quitter les sentiers battus bien tracés à l'avance et sans aspérités issus des théories liées au capital social pour t'engager dans ce qui, à cette époque, avait plus l'air d'un chemin de traverse, voire de ce que Cyrulnik (2003) appellerait "un sentier de chèvre " par opposition à l'idée d'autoroute lisse et balisée. Et c'est à ce moment que tu as commencé à t'intéresser aux « processus relationnels ».

\section{Appel à la vigilance sur la route ethnographique}

Dans le cadre de cette première rencontre, la vigilance ethnographique a été d'accepter de perdre le nord, de ne plus se laisser guider par les premières perspectives, mais plutôt de les questionner et d'intégrer de nouveaux éclairages pour que l'objet théorique puisse se transformer à la lumière des découvertes issues des observations. La vigilance ethnographique a ainsi permis de passer d'un objet théorique mesurable, défini, que Latour (1999) qualifierait de « chauve » (les relations sociales à travers le concept de capital social) à un objet 
complexe, aux contours brouillés, «échevelé » dirait l'auteur (les processus relationnels).

\section{La rencontre avec les acteurs du terrain}

Arpenter un terrain, c'est avant tout faire des rencontres avec des acteurs personnalisés (dans le sens d'incarnés), qui font avancer la recherche au travers d'échanges dont l'importance peut n'apparaître que tardivement. Cette deuxième rencontre concerne cette variété d'acteurs rencontrés, cette pluralité d'épreuves et d'expériences avec ces interlocuteurs privilégiés. Ici, la suspension du discours institutionnel intégré par la plupart des acteurs scolaires (direction, enseignants, spécialistes) a permis d'adopter une perspective compréhensive à l'égard du point de vue d'autres acteurs, afin de pouvoir mieux tenir compte des stratégies mises en place par les élèves quand ils rencontrent des difficultés dans leur parcours éducatif, mais également du sens qu'ils accordent aux différentes situations éducatives.

\section{À rebrousse-chemin}

Marjorie : Débarrassée de mon cadre théorique trop rigide, je me suis mise à observer les différents processus relationnels qui se tissent entre les élèves et leur environnement. Je me suis notamment rendu compte que l'école mettait en place toutes sortes de ressources pour les élèves quand ils rencontraient des difficultés scolaires (orthopédagogue, infirmier, conseiller en orientation, psychologue, etc.), et que la grande majorité d'entre eux ne les mobilisaient pas. La raison principale invoquée par les enseignants, la direction, les parents (et même les élèves!) était leur absence de motivation. Ainsi, les élèves n'acceptaient pas l'aide de l'enseignant, des animateurs, ne se tournaient pas vers le conseiller en orientation parce que, selon les dires du personnel scolaire, ils n'étaient pas motivés ou engagés ou encore parce qu'ils ne voulaient pas faire d'efforts.

Joëlle : Becker (2002) qui, on le devine, a beaucoup influencé ma façon de faire de la recherche, propose de suspendre les jugements reçus lorsqu'on aborde un terrain. Et derrière l'interprétation psychologique de la motivation ou de l'engagement qui, comme le dirait Mehan (1996), situe l'explication des conduites «sous la peau et entre les oreilles », je t'ai invitée à adopter une autre posture sociologique (ou posture beckerienne) et à aborder l'engagement des élèves sous l'angle de la coordination de leurs activités. Il s'agissait de ne plus voir la motivation comme «encapsulée » dans la tête des élèves, mais de chercher à saisir les projets qu'ils se donnent - et non ceux que l'institution scolaire a définis pour eux - en étant attentive aux interactions qu'ils ont avec le monde dont ils font l'expérience. 


\section{Aire de repos et de réflexion}

\section{Marjorie}

La perspective interactionniste m'a permis d'opérer un changement de point de vue et de dépasser ce regard psychologisant qui qualifie les conduites qui s'écartent de la norme (ici la norme scolaire) comme «déviantes » (Becker, 1963/1985). Et, à l'instar de Becker (1982/2006) et Morrissette (2010), j'ai commencé à appréhender les phénomènes comme le résultat d'une action collective impliquant des activités complexes issues d'une chaîne de coopération ${ }^{6}$. J'ai ainsi réalisé que les activités des uns étaient liées et affectaient les activités des autres, et que l'activation des relations sociales par les élèves était le résultat des interactions entre l'ensemble des acteurs scolaires. Les relations entre les élèves et les acteurs scolaires devenaient ainsi plus riches et enchevêtrées que le discours sur la motivation scolaire ne le donnait à voir.

J'ai donc pris mes distances avec le discours dominant et institutionnalisé des principaux acteurs scolaires qui avaient intégré le discours de l'institution. En observant attentivement, j'ai remarqué que beaucoup d'élèves étaient engagés ou s'investissaient dans d'autres formes d'activités que les formes académiques. Certains s'impliquaient dans des sports comme le basketball, d'autres faisaient du bénévolat à la Maison des jeunes, dans les églises. D'aucuns, enfin, aidaient ponctuellement les employés du Café Jeunesse.

Joëlle : Cette coopération que tu décris entre ces différents acteurs est en lien avec les dimensions collectives et dynamique des systèmes de négociation de Becker (1982/2006), qui font référence à différents «mondes » constitués d'un grand nombre d'acteurs qui, interagissant entre eux, structurent les actions de ceux qui y prennent part. Derrière la notion de « conventions », Becker relève l'ensemble des manières de faire qui facilite la coordination des actions au sein d'un monde sur la base de « compréhensions partagées ». C'est à partir de cette vision de la vie sociale qu'il suggère, dans ses ficelles du métier (2002), d'anticiper toutes les personnes qui peuvent être concernées par un objet de recherche, même celles qui en semblent très loin a priori, et de remonter chacun de ces liens pour voir en quoi ces personnes sont liées à l'action collective. De même que dans son livre Les mondes de l'art (1982/2006) dans lequel il montre comment l'art est collectif et se construit à travers les activités coordonnées d'un ensemble d'acteurs, tu voyais bien que les jeunes sur ton terrain de recherche développaient des stratégies dans leurs échanges avec différents acteurs qui gravitaient autour de l'école. Ils avaient leur propre manière d'interagir, en fonction de leurs enjeux et intérêts propres, en ne mettant pas (nécessairement) les pieds dans les sentiers que l'on avait tracés pour eux. C'est à ce moment que tu as commencé à regarder comment les élèves eux-mêmes définissaient la 
situation et que tu as franchi une étape cruciale dans ton cheminement doctoral en t'intéressant (finalement!) à leur propre idée de la « réussite».

\section{Déviation}

Marjorie : J'ai en effet compris que les élèves composaient leur propre «définition de la situation» (Thomas, 1923), c'est-à-dire leur propre engagement subjectif dans leurs actions et leur définition de cet engagement. Tout d'abord, j'ai réalisé que les élèves négociaient le sens dans leur interaction et pouvaient ainsi prêter à certains acteurs des rôles et des fonctions qui ne leur étaient pas initialement dévolus. Par exemple, un élève s'adressait à un intervenant pour faire son curriculum vitae alors que cela faisait partie des rôles du conseiller en orientation; un autre demandait à un animateur de le conseiller dans la dispute juridique qui opposait ses parents. Ainsi, ils activaient des relations parallèles, en substitution à celles offertes par l'école, d'autres fois en complémentarité. Enfin, certains élèves utilisaient ces ressources pour mieux cerner celles offertes par l'école. Cette nouvelle perspective non déficitaire du point de vue des élèves, dans la mesure où ils étaient capables d'activer leurs relations sociales selon leurs propres projets, m'a ainsi conduite sur un chemin secondaire: les stratégies parallèles que les élèves développaient quand ils rencontraient des difficultés dans leur parcours.

$\mathrm{Au}$ fil de la route, j'ai réalisé que cette négociation de sens ne se limitait pas aux seuls rôles et fonctions des acteurs, mais que les élèves avaient également des définitions différentes sur l'ensemble des situations scolaires. Ainsi, la «réussite» scolaire, au sens entendu par l'école, prenait des formes différentes selon les élèves : un élève parlait du fait de ne pas avoir de casier judiciaire, un autre de ne pas finir comme itinérant. C'est donc le choix de m'intéresser aux significations subjectives derrière les conduites qui m'a amenée à privilégier la notion de «s'en sortir» à la notion de réussite scolaire, laquelle véhicule la vision de l'institution et restreint l'idée de dépasser les obstacles à la performance scolaire.

\section{Appel à la vigilance sur la route ethnographique}

Au cours de cette deuxième rencontre, la vigilance ethnographique a consisté à prêter attention aux contradictions, à remettre en question et suspendre les jugements reçus, pour voir les choses autrement. Il a fallu aller au-delà des acteurs qui (re)produisent le discours institutionnel, en vue de s'ouvrir à d'autres logiques, d'autres processus, et intégrer ces nouvelles perspectives pour que l'objet empirique se construise à travers cette contradiction. Cette vigilance a ainsi permis de passer d'un objet abordé dans une perspective normative et déterminée (la motivation et l'engagement des élèves) qui repose sur un modèle d'acteur plus ou moins passif, à un objet abordé dans une perspective 
compréhensive et interactionniste (la négociation des processus relationnels) qui repose sur un modèle d'acteur compétent, engagé dans des projets particuliers variés.

La rencontre avec soi comme ethnographe et avec les participants à la recherche

Le parcours s'achève sur cette dernière rencontre, celle qui est la plus complexe et la plus évolutive: la rencontre avec soi en tant qu'ethnographe, mais également avec les participants comme acteurs de la recherche. La distanciation avec les postures prescrites a laissé place à une négociation des rôles au plan des postures du chercheur, mais aussi, et surtout, des participants à la recherche. Cette avancée dans l'indétermination des rôles a permis de mieux saisir les opportunités du terrain.

À rebrousse-chemin

Marjorie : Comme nous l'avons déjà mentionné, on devient ethnographe en « vivant » son terrain selon une démarche incertaine. Or, quand j'ai commencé à arpenter mon terrain, mon réflexe premier a été de chercher des directives, des règles de conduite (à cause d'une forme de candeur dans le regard). Je me suis donc raccrochée aux canons de l'ethnographie et aux différents guides méthodologiques comme celui de Beaud et Weber (2003) ou encore au manuel d'ethnographie de Mauss (1926). J'ai notamment compris qu'il existe plusieurs postures d'observation auxquelles la grande majorité des auteurs se réfèrent en lien avec la typologie classique des rôles d'Adler et Adler (1987).

Joëlle : Selon cette typologique, l'ethnographe peut ainsi endosser plusieurs rôles qui correspondent, selon Lapassade (n.d.), à l'appartenance au terrain, soit les différentes formes de rapport que le chercheur y entretient. Il s'agit en fait des divers degrés de révélation (observation masquée ou ouverte), de distanciation (observation participante périphérique, active ou complète) et de compréhension (observation non participante ou observation participante) du terrain. Si je me rappelle bien, et comme tu t'y étais engagée dans ton échéancier avant même ton entrée officielle sur le terrain, tu avais retenu une certaine posture en lien avec les «outils» de collecte sélectionnés : une posture d'observation périphérique, selon laquelle le chercheur participe aux actions comme "membre » sans être au centre des activités et n'endosse pas ainsi de rôle significatif dans la situation à l'étude (Lapassade, n.d.).

Aire de repos et de réflexion

Marjorie: D'emblée j'ai pris conscience que cette posture d'observateur périphérique ne collait pas avec mon terrain. Cela créait une certaine distance avec certains élèves, ceux-là mêmes qui n'arrivaient pas à me catégoriser, qui ne 
comprenaient pas l'intérêt d'une recherche doctorale ou encore qui essayaient de me coller une étiquette d'enseignante, de bénévole, d'intervenante, voire «d'espionne», se méfiant de moi. À cela s'ajoute le fait que cette posture m'empêchait de saisir certaines opportunités qui se présentaient puisque je restais une observatrice externe à l'action. De fait, je ne pouvais pas prendre part aux activités qui sollicitaient une observation participante active ou centrale (coach de hockey, surveillante, accompagnatrice de l'aide aux devoirs) et les fonctions inhérentes à ces activités ne cadraient plus avec la posture d'observateur périphérique prescrite.

Joëlle : Cette question a d'ailleurs constitué le premier pas d'une réflexion sur l'aspect quelque peu figé des postures d'observation prescrites par Adler et Adler (1987). Un peu à l'instar d'Hopwood (2007), tu t'es rendu compte que la posture de l'ethnographe n'était pas définitive, que les choix méthodologiques n'étaient pas arrêtés, mais, au contraire, qu'ils pouvaient se modifier à tout instant, en fonction des interactions avec les acteurs du terrain. Tu as donc commencé à te détacher de ton cadre méthodologique initial afin de pouvoir davantage coller au terrain et en saisir les opportunités. Pour le dire autrement, ton degré d'implication dans la recherche n'était plus scénarisé. Cela t'a conduite à un questionnement plus global et j'ai été témoin de tes interrogations sur les différents rôles que l'on attribue au chercheur. L'institution formule des attentes en demandant au chercheur de réfléchir à son engagement sur le terrain et de se projeter (ce que tu montrais dans ton échéancier), mais les participants d'une recherche manifestent eux aussi des attentes en essayant de ranger le chercheur dans des catégories.

\section{Déviation}

Marjorie : J'ai donc opté pour ce qu'Adler et Adler (1987) de même que Lapassade (n.d.) nomment «observation complète par opportunité », selon laquelle le chercheur exploite les circonstances, misant sur son statut déjà acquis. Dans mon cas, je dirais qu'il s'agissait davantage d'une observation par opportunité négociée avec le terrain. Cette posture tout en flexibilité m'a permis de changer les rôles au cours de mon ethnographie et de varier les points de vue sur mon objet de recherche. J'ai ainsi pu prendre part à des observations de manière externe (observer les élèves lors de mes balades dans les couloirs de l'école ou de mes tours de surveillance dans les couloirs, surveiller les comités d'élèves) ou interne (observatrice aux rencontres des comités). Je me suis également impliquée de manière périphérique (participante aux ateliers, aux différents jeux), active (j'ai mis en place l'aide aux devoirs) et parfois complète (quand certains élèves ne savaient pas que je faisais une recherche). D'observatrice, je suis devenue moi-même, par moments, un objet de ma 
recherche puisque les jeunes sollicitaient également mon aide. De fait, il a fallu que je m'analyse moi-même comme une ressource que les élèves mobilisaient ou non, et que j'essaie de comprendre le sens qu'ils accordaient à cette relation parmi d'autres.

Joëlle : Et cette négociation des rôles concernait également les élèves puisqu'il s'agit d'une interaction (on est interactionniste ou on ne l'est pas!). Ici, ton questionnement de départ a évolué autour de la notion de réflexivité des acteurs en situation (Morrissette \& Guignon, 2006). D'autant plus que le recours à Giddens (1984) te permettait d'appréhender l'élève comme un acteur compétent (capable d'activer ses relations sociales) et réflexif (capable de rendre compte de cette activation) en interaction avec son environnement. Il est effectivement inconcevable d'occulter le point de vue des acteurs si l'on veut comprendre leur expérience, bien qu'il faille retenir la nuance apportée par Giddens, pour qui la réflexivité des acteurs présente des limites en raison des conséquences non intentionnelles qu'entraînent leurs actions.

Marjorie : C'est vrai! Et les traditions participatives ont infléchi ma conception de l'implication des acteurs. Il s'agissait dès lors de mettre à profit leurs compétences parfois énoncées sous la forme de critères de rigueur chez Savoie-Zajc (2001), notamment le critère de respect des valeurs et des principes démocratiques (ou respect du monde empirique, chez Woods, 1992). Je développais un attrait pour ces recherches qui affirment que les chercheurs travaillent non plus sur, mais avec les acteurs, et favorisent l'implication des participants à la recherche (Morrissette \& Guignon, 2006; Savoie-Zajc, 2001). Du rôle de simples participants, les élèves sont progressivement devenus des acteurs de ma recherche. Les entretiens de groupe ont pris la forme d'analyses de groupe qui sollicitent la réflexivité des personnes concernées par le problème étudié (Van Campenhoud, Franssen, \& Cantelli, 2009). J'ai donc commencé à soumettre aux élèves les résultats de mes premières analyses pour qu'ils puissent exprimer leurs interprétations sur les processus relationnels en œuvre. En appréhendant ainsi les élèves comme des acteurs sociaux, je pouvais désormais confronter nos analyses respectives et développer, en filigrane, cette idée de rapprochement, de négociation entre nos deux mondes.

\section{Appel à la vigilance sur la route ethnographique}

Cette posture flexible et négociée avec le terrain ouvrait une route moins balisée que celle proposée par les méthodes de recherche plus conventionnelles qui invitent à retenir et privilégier une posture d'observation sur un temps précis, figeant en quelque sorte la relation du chercheur avec le terrain. Dans ce cas, faire preuve de vigilance ethnographique, c'était accepter de se départir de ces orientations méthodologiques fixées, voire réifiées, et de se fier au sens de 
l'orientation, à cette boussole qui se constituait au fur et à mesure des avancées - et parfois des reculs! - sur le terrain afin de renégocier les rôles du chercheur et des participants. Une fois acceptée la distanciation avec le scénario méthodologique initial, les évènements, les rencontres et surtout l'ajustement continu avec le terrain ont ensuite balisé les formes de l'implication de part et d'autre.

\section{Conclusion: vers une construction théorique de la vigilance ethnographique}

Dans cet article, nous avons théorisé l'idée de vigilance par analogie avec l'idée d'itinéraire chaotique, une errance temporelle théorique et empirique, jalonnée de rencontres impromptues et influentes, de discussions imprévues qui ont conduit une doctorante à renégocier ses choix initiaux. La vigilance s'est ainsi définie à travers plusieurs manifestations, entre le renoncement de la voie toute balisée à l'acceptation de prendre des chemins de traverse, de se perdre, de se réorienter et de redéfinir la trajectoire. La vigilance ethnographique, c'est donc être attentif à ce qui se passe quand on saute vers l'inconnu, mais c'est aussi (et surtout) savoir composer avec les obstacles qui se présentent. À l'instar de Giddens, il s'agit de ne plus les voir comme des contraintes, mais davantage comme des ressources pour l'action. Chacun de ces obstacles recouvre des tâtonnements successifs, telle une composition perpétuelle avec le terrain qui permet d'intégrer de nouvelles perspectives théoriques/épistémologiques afin que l'objet se construise et se (trans)forme.

En tant qu'ethnographe en herbe, il a pourtant fallu s'entêter à créer les conditions de cette négociation, car les exigences universitaires, orientées vers l'efficience, ne laissent que peu de place à l'errance ethnographique. Confronté à un cadre universitaire québécois contraignant, l'étudiant en science de l'éducation qui privilégie l'ethnographie doit se créer une marge de manœuvre en repoussant ou contournant certaines exigences officielles de son institution. À l'image du métier d'élève tel qu'envisagé par Perrenoud (2004), le doctorant en vient à infléchir les règles pour survivre à un contexte trop contraignant pour l'ethnographie; cette méthode d'enquête requiert souplesse et flexibilité et également un rapport différent au terrain et aux acteurs sociaux afin de laisser l'objet se (re)construire au fil de l'épreuve empirique des conceptualisations.

Reste la question de savoir comment rendre compte d'une telle démarche, marquée par ces changements de direction. Au passage difficile entre l'institution et le terrain succède un passage de retour vers l'institution tout aussi délicat à négocier. Une des questions de l'appel de textes était de savoir « comment produire la preuve » ethnographique. En d'autres termes, il s'agissait de s'interroger sur les façons de rendre compte de cette démarche non linéaire 
alors que les normes dominantes dans la communauté scientifique privilégient un mode scriptural linéaire au langage et au monde, et que l'ethnographie met l'accent sur la transparence et le processus de recherche. Â juste titre, Demazière (2008) déplore que les propos méthodologiques des recherches soient trop aplatis, finis, lisses, et mettent de l'avant un produit bien emballé qui ne reflète pas les aspérités du terrain. À l'opposé, on peut se demander jusqu'où aller pour rendre compte des tâtonnements successifs. Quelle place doit-on accorder aux errances ethnographiques? Faut-il retranscrire toutes les pistes, même celles qui ne se sont pas révélées fécondes? Ces questions se posent avec d'autant plus d'acuité que le format de thèse est particulièrement standardisé et avalisé par l'institution. Dans le cadre de cette recherche, on peut par exemple se demander si et comment témoigner du cadre théorique initial, forgé autour du capital social, sachant le rôle qu'il peut avoir joué quelque part dans la recherche, telle une tache aveugle (von Foerster, 1988).

Si la réflexion sur ce plan est encore à poursuivre, un premier pas vers la réponse peut être franchi grâce à Degand (2012). L'auteure propose une structure de thèse moins formatée par les exigences académiques et plus respectueuse du terrain. Il nous semble en effet que commencer une thèse par la méthodologie permet de respecter la démarche ethnographique, telle qu'elle a eu lieu. La problématique peut ainsi se construire au travers du récit méthodologique, en fonction du processus de construction de l'objet sur le terrain. Finalement, cela confère une visibilité et une légitimité accrues aux tâtonnements successifs et aux errances ininterrompues afin de rendre in fine le ballotage favorable à l'ethnographe.

\section{Notes}

1 L'usage des guillemets souligne une distance prise avec l'utilisation du terme défavorisé tel qu'employé dans la littérature sur les inégalités sociales. Ce terme renvoie à des inégalités économiques, culturelles et symboliques.

2 Tillard (2011) propose une division de l'ethnographie selon deux autres temps : le temps de l'observation et le temps de l'écriture. Cependant, dans le cadre de cet article, nous considérons que ces deux temps font partie du temps ethnographique que nous appréhendons en comparaison avec le temps de l'institution.

3 Poupart, J. (2011, Février). La tradition de Chicago. Conférence donnée dans le cadre du cours ETA-6507, Démarches d'investigation des pratiques professionnelles (responsable J. Morrissette), Faculté des sciences de l'éducation, Université de Montréal, Montréal. 
${ }^{4}$ Pour illustrer le propos, les élèves haïtiens utilisaient une expression récurrente : manger $d u$ bâton, qui signifie que l'élève allait « se faire gronder vigoureusement, voire frapper » une fois rentré chez lui.

${ }^{5}$ Cet article ne peut présenter de manière exhaustive la théorie de Giddens. Les lecteurs se réfèreront à son ouvrage (1984) ou à ceux d'autres auteurs qui en traitent tels que van Haecht (1998).

${ }^{6}$ Comme le précisent de Queiroz et Ziolkowski (1997), la notion de coopération n’est pas employée ici dans un sens moral, mais renvoie aux divers modes d'interaction négociés pris en compte dans l'interactionnisme symbolique (confrontation, participation, résistance, etc.).

\section{Références}

Adler, P., \& Adler, P. (1987). Active membership. Membership roles in field research. London : Sage.

Barakett, J., \& Cleghorn, A. (2008). Sociology of education: an introductory view from Canada ( $2 \mathrm{e}$ éd.). Toronto : Pearson.

Baudelot, C., \& Establet, R. (1971). L'école capitaliste en France. Paris : Maspero.

Beaud, S., \& Weber, F. (2003). Guide de l'enquête de terrain. Paris : La Découverte.

Becker, H. S. (1985). Outsiders. Études de sociologie de la déviance. Paris: A.M. Métailié. (Ouvrage original publié en 1963).

Becker, H. S. (2002). Les ficelles du métier. Paris : La Découverte.

Becker, H. S. (2006). Les mondes de l'art. Paris: Flammarion. (Ouvrage original publié en 1982).

Becker, H. S. (2011, Mars). Quand les chercheurs n'osent plus chercher. Le monde diplomatique, 14-15.

Blumer, H. (1969). Symbolic interactionism: perspective and method. Englewood Cliffs, NJ : Prentice-Hall.

Boudon, R. (1973). L'inégalité des chances; la mobilité sociale dans les sociétés industrielles. Paris : Armand Colin.

Bourdieu, P., \& Passeron, J.- C. (1970). La reproduction: éléments pour une théorie du système d'enseignement. Paris : Minuit.

Chapoulie, J. M. (2001). La tradition sociologique de Chicago, 1892-1961. Paris : Seuil. 
Coleman, J. S. (1988). Social capital in the creation of human capital. The American Journal of Sociology, 94(1), 95-120.

Coulon, A. (1997). Le métier d'étudiant. L'entrée dans la vie universitaire. Paris : Presses universitaires de France.

Cyrulnik, B. (2003). Les sentiers de chèvres et l'autoroute. Dans V. Duclert (Éd.), Quel avenir pour la recherche (pp. 70-79). Paris : Flammarion.

Degand, A. (2012). Le journalisme face au web : reconfiguration des pratiques et des représentations professionnelles (Thèse de doctorat inédite). Université Catholique de Louvain, Belgique.

Demazière, D. (2008). L'entretien biographique comme interaction. Négociations, contre-interprétations, ajustement de sens. Langage et société, 123, 15-35.

Derouet, J. L., \& Henriot, A. (1987). Approches ethnographiques en sociologie de l'éducation: l'école et la communauté, l'établissement scolaire et la classe. Notes de synthèse. Revue française de pédagogie,78, 73-108.

Dika, S. L, \& Singh, K. (2002). Applications of social capital in educational literature : a critical synthesis. Review of Educational Research, 72(1), 3160.

Fortin, S., \& Houssa, E. (2012). L'ethnographie postmoderne comme posture de recherche : une fiction en quatre actes. Recherches qualitatives, 31(2), 5278.

Giddens, A. (1984). The constitution of society. Outline of the theory of structuration. Cambridge : Cambridge University Press.

Glaser, B. G., \& Strauss, A. L. (1967). The discovery of grounded theory: strategies for qualitative research. Chicago, IL : Aldine.

Hopwood, N. (2007). Researcher roles in a school-based ethnography. Dans G. Walford (Éd.), Studies in educational ethnography: methodological developments in ethnography (pp. 51-68). Oxford : Elsevier.

Lapassade, G. (n.d.). La méthode ethnographique : l'observation participante. Repéré à http://www.vadeker.net/corpus/lapassade/ethngr1.htm.

Latour, B. (1999). Politiques de la nature. Comment faire entrer les sciences en démocratie? Paris : La Découverte.

Malinowski, B. (1922). Les argonautes du pacifique occidental. Paris : Gallimard.

Mauss, M. (1926). Manuel d'ethnographie. Paris : Payot. 
Mehan, H. (1996). Beneath the skin and between the ears : a case study in the politics of representation. Dans S. Chaiklin, \& J. Lave (Éds), Understanding practice: perspective on activity and context (pp. 241-268). New York, NY : Cambridge University Press.

Morrissette, J. (2010). Une perspective interactionniste. SociologieS. Repéré à $\mathrm{http} / / /$ sociologies.revues.org/3028.

Morrissette, J., \& Guignon, S. (2006). Quand les acteurs mettent en mots leur expérience. Recherches qualitatives, 26 (2), 19-38.

Morrissette, J., Guignon, S., \& Demazière, D. (Éds). (2011). De l'usage des perspectives interactionnistes en recherche. Recherches qualitatives, 30(1).

Parsons, T. (1959). The school class as a social system : some of its functions in American society. Harvard Educational Review, 29(5), 297-318.

Perrenoud, P. (2004). Métier d'élève et sens du travail scolaire. Paris : Éditions sociales françaises.

Poupart, J. (2011, Février). La tradition de Chicago. Communication présentée dans le cadre du cours ETA-6507-Démarches d'investigation des pratiques professionnelles. Faculté des sciences de l'éducation. Université de Montréal, Montréal.

Queiroz, J.- M., \& Ziolkowski, M. (1997). L'interactionnisme symbolique. Rennes : Presses universitaires de Rennes.

Savoie-Zajc, L. (2001). La recherche action en éducation: ses cadres épistémologiques, sa pertinence, ses limites. Dans M. Anadón (Éd.), Nouvelles dynamiques de recherche en éducation (pp. 15-49). Québec: Presses de l'Université Laval.

Soukup, C. (2012). The postmodern ethnographic flaneur and the study of hyper-mediated everyday life. Journal of Contemporary Ethnography, 42(2), 226-254.

Thomas, W. I. (1923). The unadjusted girl with cases and standpoint for behavior analysis. Boston, MA : Little Brown Company.

Tillard, B. (2011). Temps d'observation ethnographique et temps d'écriture. Les sciences de l'éducation pour l'ère nouvelle, 44, 33-47.

Van Campenhoudt, L., Franssen, A., \& Cantelli, F. (2009). La méthode d'analyse en groupe. SociologieS. Repéré à http://sociologies.revues.org/2968

Van Haecht, A. (1998). L'école à l'épreuve de la sociologie. Questions à la sociologie de l'éducation ( $2^{\mathrm{e}}$ éd.). Bruxelles : De Boeck. 
Vienne, P. (2005). Mais qui a peur de l'ethnographie scolaire? Éducation et sociétés, 16, 177-192.

Von Foerster, H. (1988). La construction d'une réalité. Dans P. Watzlawick (Éd.), L'invention de la réalité. Contributions au constructivisme (pp. 4569). Paris : Seuil.

Woods, P. (1990). Ethnographie de l'école. Paris : Armand Collin.

Woods, P. (1992). Symbolic interactionism : theory and method. Dans M. D. Le Compte, W. L. Millroy, \& J. Preissle (Éds), The handbook of qualitative research in education (pp. 337-404). New York, NY : Academic Press.

Yan, W. (1999). Successful African American students : the role of parental involvement. Journal of Negro Education, 68(1), 5-22.

Zhang, X. Y., DeBlois, L., Kamanzi, C., \& Deniger, M.- A. (2008). A theory of success for disadvantaged children : re-conceptualisation of social capital in the light of resilience. Alberta Journal of Educational Research, 54(1), 97112 .

Marjorie Vidal est doctorante en sciences de l'éducation à l'Université de Montréal. L'exclusion sociale et le décrochage scolaire sont au cour des thématiques qui l'intéressent. Dans sa thèse, elle appréhende les relations sociales comme des leviers que les élèves mobilisent pour "s'en sortir » quand ils rencontrent des difficultés dans leurs parcours éducatifs. Son cheminement l'a conduite à s'intéresser à l'ethnographie et à l'interactionnisme symbolique pour leurs apports à l'approche d'intervention et de justice sociale.

Joëlle Morrissette est professeure adjointe à la Faculté des sciences de l'éducation de l'Université de Montréal. Elle s'investit particulièrement en recherche méthodologique, selon une perspective interactionniste qui conduit à saisir, de manière compréhensive, les significations des pratiques professionnelles pour ceux et celles qui les mettent en œuvre, et à repérer les processus d'ajustement et de négociation au principe de leur régulation. Ses enseignements aux $2^{e}$ et $3^{e}$ cycles sont centrés sur différentes traditions de recherche qualitative. 